ARTIGO

\title{
CIENCIA DE LA INFORMACIÓN Y CIENCIA DE LA COMUNICACIÓN: “otro dialogo interdisciplinar ${ }^{i}$
}

\author{
INFORMATION SCIENCE AND COMMUNICATION SCIENCE: "another one dialogue \\ interdisciplinary"
}

Radamés Linares Columbié ${ }^{1}$

\author{
${ }^{1}$ Doutor em Ciências da Comunicação pela \\ Universidade de la Habana, (CUBA). \\ E-mail: radames@fcom.uh.cu
}

\section{A ACESSO ABERTO}

Copyright: Esta obra está licenciada com uma Licença Creative Commons Atribuição 4.0 Internacional. $(\mathrm{cc}) \mathrm{EY}$

Conflito de interesses: 0 autor declara que não há conflito de interesses.

Financiamento: Não há.

Declaração de Disponibilidade dos dados: Todos os dados relevantes estão disponíveis neste artigo.

Recebido em: 20/09/2018.

Revisado em: 01/10/2018.

Aceito em: 10/10/2018.

Como citar este artigo:

LINARES COLUMBIÉ, Radamés. Ciencia de la Información y Ciencia de la Comunicación: "otro dialogo interdisciplinar". Informação em Pauta, Fortaleza, v. 3, número especial, p. 30-46, nov. 2018. DOI: https://doi.org/10.32810/25253468.ip.v3iEspecial.2018.39712.30-46.

\section{RESUMEN}

Se examina la diversidad de discursos que expresan los procesos de organización, producción y difusión del conocimiento. Establece los rasgos identificadores de la Ciencia de la Información y la Ciencia de la Comunicación. Discute las propuestas de interdisciplinariedad que caracterizan a los cuerpos de conocimiento que se estudian. Precisa el carácter de los diálogos existente entre la Ciencia de la Información y la Ciencia de la Comunicación. Incluye referencias bibliográficas y una propuesta de conclusiones).

Palabras-clave: Ciencia de la Información. Ciencia de la Comunicación. Interdisciplinariedad.

\section{ABSTRACT}

The diversity of discourses that the processes of organization, production and diffusion of knowledge express is examined. Establish features identifiers of the Information Science and Communication Science. You discuss interdisciplinary's proposals that characterize the bodies of knowledge that are studied. The character needs the existent dialogues between of the Information Science and Communication Science. You include bibliographic references and a proposal of findings.

Keywords: Information Science. Communication Science. Interdisciplinary. 


\section{INTRODUCCIÓN}

A lo largo de la historia, los campos de conocimiento han ido transitando, primero, por un determinado esfuerzo por delimitar sus fronteras en relación con otras ramas colindantes, y; segundo, por su apertura a la interacción con otros campos, algunos más cercanos que otros.

Estos procesos instauraron formas específicas de organización y producción del conocimiento en diversas ramas denominadas comúnmente como: disciplinas, multidisciplinas, interdisciplinas y transdisciplinas. Los campos de la información y de la comunicación, al igual que otros campos de conocimiento han sido caracterizados en una u otra variante disciplinar; pese a ello, la tipificación como interdisciplina es de una relevancia particular, no solo al interior de uno u otro espacio, sino también en las interacciones existentes entre estos espacios.

La etiqueta "interdisciplina" tiene varias facetas, entre otras, se destacan: la referida a la educación, donde es entendida como una forma de estructurar los programas de estudios o currículos y otra que remite a la producción y organización del conocimiento que se genera en uno u otro campo

$\mathrm{Al}$ igual que otros movimientos intelectuales, la interdisciplinariedad tiene su historia, instituciones e ideologías. Un instante sobresaliente del quehacer interdisciplinar se ubica después de la 2da posguerra mundial, donde la expresión ideológica más notable lo fue la célebre "revolución de mayo de 1968", movimiento político y social que impugno el modelo disciplinar vigente en las universidades francesas y postulo otras variantes para la transmisión y organización de los saberes.

En rigor, uno de los factores impulsores del nacimiento del movimiento interdisciplinar gira en torno a lo que se denomina usualmente como la "crisis de las disciplinas", estas últimas como variantes organizativa y productora de conocimientos tuvieron en el siglo XIX su máximo esplendor y agotamiento; generando una contradicción para el desarrollo del conocimiento científico y su necesidad de crecimiento, haciéndose evidente la necesidad de síntesis, con su consiguiente interacción e integración como formas necesaria para asumir la complejidad de la realidade.

De otra parte, el propio desarrollo del quehacer interdisciplinar impulso su institucionalización con el empuje que recibió de parte de instituciones como la UNESCO 
(Organización de Naciones Unidas para la educación, la ciencia y la cultura) y la OCDE (Organización para la cooperación y el desarrollo); así como con el surgimiento de programas interdisciplinares en varias universidades del mundo.

Por último, es observable que los procesos de construcción e identificación de la Ciencia de la Información y la Ciencia de la Comunicación como campos de conocimientos están especialmente insertados en las dinámicas, antes mencionadas. En consecuencia, este texto, busca examinar los elementos más notables que permiten delimitar el carácter de las relaciones e interacciones existentes entre la Ciencia de la Información y la Ciencia de la Comunicación.

\section{LOS CAMPOS DE CONOCIMIENTOS}

El conocimiento académico ha organizado y estructurado su producción de conocimientos a lo largo del tiempo a través de las denominadas: Disciplinas, Multidisciplinas, Interdisciplinas y Transdisciplinas.

El termino disciplina tiene más de un significado, tanto en el lenguaje común como en el académico. Comúnmente se entiende como una regla de conducta que un individuo se impone o las circunstancias le exigen; en el universo académico se asume como un dominio particular de conocimiento -entendida como disciplina científica (rama del conocimiento) o disciplina escolar (componente curricular).

Los orígenes de las disciplinas son bastante lejanos. El siglo XIX se considera significativo en la historia de las disciplinas; es la época de un sustantivo auge de las ciencias naturales y del surgimiento de las ciencias sociales. Desde ese momento, la particularización y segmentación del conocimiento se establece como el procedimiento organizativo del saber, el cual permite la obtención de conocimientos particulares. Así, se comienza a entender que una disciplina es un segmento de conocimiento, del conjunto de conocimientos de la humanidad, convertido en singular, particular, distinto. Fragmentar, parcelar el examen de la realidad es el rasgo que por excelencia distingue a las disciplinas. Además, en ellas habría que incluir a una comunidad, una determinada práctica, una tradición, un conjunto particular de valores y creencias, un dominio, una modalidad de investigación y una estructura conceptual. 
La explosión disciplinaria - que es la tónica del conocimiento científico en los siglos XIX y XX- es expresión de las plataformas y concepciones propias de la modernidad, particularmente, concepciones filosóficas como: el racionalismo, el empirismo y el positivismo. La perspectiva analítica de la realidad es uno de los soportes del pensamiento racionalista, y uno de los fundamentos de la propuesta disciplinar y su fragmentación y parcelación de los diversos dominios de la realidad; algo similar ocurre con el positivismo y su propuesta objetivista, por esto cada disciplina se ocupaba sólo de su "objeto" sin contaminarse, o contaminar a los demás (POMBO, 2005).

La segunda mitad del siglo XX inicia un proceso de cambios en todos los terrenos, que va a tener también su manifestación en los espacios centrados en la organización y producción de conocimientos en todos los saberes. Son estos los tiempos en que se gestan y se desarrollan las impugnaciones al denominado proyecto de la modernidad; que tiene al cuestionamiento al modelo de ciencia impulsado por la modernidad como una de las expresiones criticas ante esa visión.

"Fronteras", "limites", “autonomía": rasgos típicos del esquema disciplinar, comienzan a mostrar su incapacidad para responder a problemas y circunstancias de una época nueva. Algo similar sucede con los basamentos teorico-filosoficos de la opción disciplinar: se desmoronan las visiones absolutas de los grandes sistemas filosóficos, se está en tiempos de rechazo a esas posiciones.

Las opciones disciplinares "multi, inter y trans" emergen en este nuevo entorno como expresión de una época nueva:

[...] Debe reconocerse que, por detrás de esas cuatro palabras multi, pluri, inter y transdisciplinariedad, hay una misma raíz - la palabra disciplina. Ella está siempre presente en cada una de ellas. Lo que nos permite concluir que todas ellas tratan de cualquier cosa que tiene que ver con las disciplinas. Disciplinas que pretenden unirse, multi, pluri, la idea es la misma, unir muchas, poner unas al lado de las otras. 0 entonces, articularlas, ponerlas inter, en interrelación, establecer entre ellas una acción recíproca. El sufijo trans supone un ir más allá, sobrepasar aquello que es propio de las disciplinas [...] (POMBO, 2005, p. 3).

En consecuencia, se afirma que, la Multidisciplinariedad es la visión menos controversial de la triada mencionada. Hay cierto consenso entre los expertos en considerar que esta perspectiva implica convocar a varias disciplinas, cada una de ellas con su cuerpo teórico y metodológico específico, para abordar un objeto de estudio compartido. En esta modalidad de vínculo, las disciplinas que participan no se 
modifican, ni se enriquecen al hacerlo, ya que no se producen cambios en sus esquemas conceptuales y prácticos.

De otra parte, la Interdisciplinariedad, como segundo nivel de integración disciplinar tiene un rasgo característico la diversidad de aproximaciones al significado de esta práctica y concepción; en líneas generales se tiende a considerarla con una peculiaridad: como resultado de la cooperación entre disciplinas y sus interacciones (esta puede ir desde una simple comunicación de ideas hasta la mutua integración de conceptos, metodología, procedimientos, epistemología, terminología, datos y la organización de investigación y docencia en un campo suficientemente amplio); es decir, reciprocidad en los intercambios y, por consiguiente, un enriquecimiento mutuo.

La tónica de los estudios interdisciplinarios busca el desarrollo de actividades que se realizan con la cooperación e intercambio de varias disciplinas. Cada disciplina pone a disposición de las otras sus esquemas conceptuales, sometiéndolos a la asimilación y reformulación de los mismos, de los que resulta una integración diferente por esa reciprocidad en el intercambio. Es así, que:

[...] or interdisciplinariedad, deberá entonces entenderse cualquier forma de combinación entre dos o más disciplinas con vista a la comprensión de un objeto a partir de la confluencia de puntos de vista diferentes y teniendo como objetivo final la elaboración de una síntesis relativa al objeto común [...] (POMBO, 1994, p. 71).

Por último, es posible afirmar que la concepción transdisciplinar es expresión de una coyuntura epocal que se expresa en: complejidad de los problemas que surgen en el campo académico y fuera de él; excesiva especialización del saber; percepción de la existencia de múltiples verdades.

La transdisciplinariedad es una etapa superior y posterior a la interdisciplinariedad que no se limita a las interacciones o reciprocidad entre las disciplinas, sino que ubica esas relaciones o vínculos en el interior de un sistema total, sin fronteras establecidas entre las disciplinas. Con otras palabras, el enfoque transdisciplinar es la tendencia de crear puentes entre las disciplinas, un terreno común de intercambio, dialogo e integración, donde los fenómenos pueden ser asumidos desde perspectivas diferentes al mismo tiempo, generando una comprensión holística de ese fenómeno que no es posible enmarcar en ninguna disciplina.

Los estudios sistemáticos e institucionalizados de la información y la comunicación nacen y se desarrollan en la particular coyuntura de la segunda mitad del 
siglo $\mathrm{XX}$ y, en consecuencia, permeados de las nuevas formas en que se organiza la producción, organización y difusión del conocimiento, por lo cual, sus tipificaciones se adentran en el movimiento disciplinar descrito.

\section{LA CIENCIA DE LA INFORMACIÓN Y SU INTENCIÓN INTERDISCIPLINAR}

Se afirma que “[...] Aparentemente, no existe una concepción uniforme de la Ciencia de la Información. El campo parece seguir diferentes enfoques y tradiciones [...]. No obstante, todos se representan por el mismo nombre, Ciencia de la Información." (ZINS, 2007, p. 341). Este autor, también anota que circulan alrededor de decenas de explicaciones y definiciones diferentes de Ciencia de la Información, según un estudio de expertos realizado en esos años.

Esa pluralidad de explicaciones y definiciones de la Ciencia de la Información es posible agruparlas, entre otras, en varias direcciones:

- Algunos entienden que el enunciado Ciencia de la Información equivale a las denominadas actualmente Ciencias de la Información y/o Estudios de la Información (espacio que agruparía a la Bibliotecología, la Archivística y la Ciencia de la Información anglosajona);

- Otros consideran que la Ciencia de la Información tiene solo que ver con los algoritmos y las tecnologías de la información y comunicación;

- También se postula que la Ciencia de la Información equivale a la Documentación;

- Al igual que, se concibe a la Ciencia de la Información como un cuerpo de conocimiento autónomo vinculados fuertemente con otras materias informativas.

En este texto se asume que la Ciencia de la Información es un conjunto de conocimientos enmarcado en el campo de las Ciencias Sociales centrado en la producción, organización y uso de la información bajo las condiciones tecnológicas surgidas desde la 2da mitad del siglo XX y fuertemente asociada a otros campos de conocimientos informacionales, tales como: Bibliotecología, Archivística y Documentación. 
La Ciencia de Ia Información en su proceso de fundación y continuidad ha buscado legitimarse como un cuerpo de conocimiento desde múltiples aristas y perspectivas. Uno de sus rasgos notables, desde sus años fundacionales, fue su propuesta de caracterizarse como ciencia interdisciplinaria. Este autocaracterizacion de este campo de estudios está ampliamente discutida en la obra de múltiples expertos, entre otros: Borko (1968); Mikhailov, Chernyi Y Gilyarevskyi (1969); Saracevic (1992, 1996); Le Coadic (1996); Pinheiro (1999; 2006); Gonzalez De Gómez (2001); Smit; Tálamo; Kobashi (2004); Guzmán Gómez (2005).

La instalación de la Ciencia de la Información como campo de conocimiento es una de las expresiones de los cambios informativos ocurridos durante la 2 da posguerra mundial, generando situaciones informacionales que son, a su vez, el resultado de eventos ocurridos en todas las dimensiones de la sociedade.

El registro formal de la Ciencia de la Información fue expresada por Harold Borko (1922-2012) en 1968, donde expresa las concepciones esenciales de los padres fundadores de este campoii, así nos dice que la nueva materia "[...] investiga las propiedades y comportamiento de la información, las fuerzas que rigen su flujo y los medios de procesarla para su óptima accesibilidad y aprovechamiento[...]”. (BORKO, 1968, p. 2).

En el mismo texto caracteriza a la Ciencia de la Información como una “[...] ciencia interdisciplinaria derivada o que está relacionada con la Matemática, Lógica, Lingüística, Sicología, Tecnología de la computación, Investigación operacional, Artes gráficas, Comunicación y la Bibliotecología [...]”. (BORKO, 1968, p. 3).

Esta primera caracterización de esta área de estúdios:

[...] no tuvo consecuencias epistemológicas importantes para la Ciencia de la Información en estos primeros años. Tal característica fue utilizada, sobre todo, para justificar la actuación en el campo, de personas provenientes de las más variadas disciplinas científicas... Estudios más rigurosos sobre la idea de interdisciplinariedad (de la necesaria existencia de un proceso teórico y conceptual de "a dos manos" entre las disciplinas involucradas) comenzaron a diagnosticar inexistencia de prácticas interdisciplinares entre la Ciencia de la Información y las demás ciencias, en la medida en que la Ciencia de la Información "tomaba en préstamo" conceptos y métodos de otros campos, sin hacerse notar por esta [...] (ARAÚJO, 2014, p. 14).

La identificación interdisciplinaria de la Ciencia de la Información es una de las constantes de esta área. Uno de los autores que, en los finales del siglo XX, se ha detenido en examinar este tópico con más atención, es Tefko Saracevic (1930), considerado uno 
de los pensadores fundamentales de este campo de estudios y se propone en dos artículos publicados en los años 1996 y 1999 fijar su posición ante el tema de la interdisciplinariedad de la Ciencia de la Información.

Saracevic, propone que "[...] la Ciencia de la Información trata los problemas de la efectiva comunicación de conocimiento y registros de conocimiento entre seres humanos en el contexto de usos y necesidades sociales, individuales e institucionales, de información". (SARACEVIC, 1999, p. 35). De otra parte, este autor anota:

[...] Me concentrare en las relaciones interdisciplinares con dos disciplinas: la Bibliotecología y la Ciencia de la Computación. Obviamente otros campos, notablemente la Ciencia Cognitiva y la Comunicación poseen relaciones disciplinares con la Ciencia de la Información, pero las mencionadas son las más significativas y desarrolladas. (SARACEVIC, 1999, p. 35).

Las consideraciones de Harold Borko y Tefko Saracevic más allá de sus cercanías y distancias, subrayan la presencia de la Comunicación como una de las materias de relación disciplinar, coincidencia significativa de acuerdo con los propósitos de este texto. Si bien, más adelante, se volverá sobre este particular; los autores mencionados ofrecen una argumentación no precisamente amplia sobre este asunto.

\section{LA CIENCIA DE LA COMUNICACIÓN Y EL ESCENARIO INTERDISCIPLINAR}

Comunicación, Ciencia de la Comunicación, Comunicación Social y Comunicología son algunas de las denominaciones que en uno u otro momento ha recibido esta área de estudios, términos que traducen concepciones diversas en torno al carácter y propósitos de este espacio. Escapa a los objetivos de estas líneas examinar esta problemática y en consecuencia, a los efectos de estas consideraciones será Ciencia de la Comunicación la expresión que resumirá las características, historia y objetivos de este campo de conocimiento.

Los estudios sobre la Comunicación tiene una larga historia que se remonta a la antigüedad clásica y tienen al filósofo griego Aristóteles como uno de los primeros que identifica y reflexiona sobre este fenómeno al realizar sus estudios sobre retórica, donde esboza las primeras consideraciones en torno al fenómeno de la comunicación. Este acontecimiento es, sin dudas, uno de los antecedentes de la hoy llamada Ciencia de la 
Comunicación, cuya constitución formal como dominio de conocimiento es un evento que se enmarca en la 2da mitad del siglo XX y es, al igual, que la Ciencia de la Información otro de los nuevos saberes que surgen posterior a la última guerra mundial y que su aparición fue una de las expresiones de los cambios informacionales y comunicacionales que se producen en esos años (CAMARA, 2011).

La comunicación, es un término polisémico que ha dado lugar a múltiples definiciones, pero que el sentido común del campo académico asume como objeto de estudio de este campo de estudios y que en sus años iniciales se centraba exclusivamente en el universo de los medios de difusión masiva y que en nuestro tiempo va más allá de estos últimos.

Se afirma, que:

En la constitución de cualquier dominio de conocimiento, la definición de su objeto de estudios es fundacional; es en torno a un objeto propio, distinto que un nuevo dominio de conocimiento se constituye.... en líneas generales, podemos identificar dos objetos: los medios de comunicación y el proceso comunicativo. (FRANÇA, 2000).

Esta afirmación revela que es sustantivo no restringir el objeto de la Ciencia de la Comunicación al campo mediático, ya que al hacerlo se apartan las diversas practicas comunicativas que conforman la vida en sociedad y que no están necesariamente marcadas por las llamadas mediaciones tecnológicas. Por ello, se anota que:

[...] desde los inicios de los estudios sobre comunicación, diferentes autores han coincidido en que su objeto son los medios de difusión, relegando a un segundo plano otras formas de comunicación, como lo son: la comunicación interpersonal y cultural. Con la llegada de los medios de comunicación social (el cine, la radio, la televisión y la prensa) y las relaciones de poderes que se gestaban entorno a estos, provocaron que en lugar de partir de las relaciones humanas, fueran la técnica, la ingeniería y la electrónica y las poderosas empresas propietarias de los medios quienes impusieran la forma de concebir la comunicación [...]. (SALADRIGAS, 2006).

Cualquier examen de la trayectoria de la Ciencia de la Comunicación pone de manifiesto el carácter fundamentalmente social de esta disciplina, puesto que desde sus inicios, ha sido la sociología la que ha jugado un papel primordial en el abordaje de los fenómenos comunicativos. Es precisamente, este hecho y las características de su objeto de estudio lo que permite tipificar inequívocamente a este campo como perteneciente a las Ciencias Sociales y es por ello que las principales influencias que se observan en las 
teorizaciones comunicacionales provienen de corrientes principalmente sociológicas, tales como el funcionalismo, estructuralismo o la teoría crítica, entre otras.

Las características del objeto de estudio de la Ciencia de la Comunicación conducen inexorablemente a que en su estudio converjan las más diversas áreas de conocimiento y es por ello, inobjetable el carácter interdisciplinar de este dominio de conocimiento. En síntesis, es explicar el fenómeno comunicativo a partir de varias disciplinas conexas entre sí y con relaciones definidas, a fin de que sus actividades no se produzcan en forma aislada, dispersa y fraccionada. Esta caracterización como interdisciplinaria ha sido tratada y debatida por diversos autores de este campo.

\section{LOS DIÁLOGOS ENTRE LA CIENCIA DE LA INFORMACIÓN Y LA CIENCIA DE LA COMUNICACIÓN}

Cualquier aproximación al examen de las proximidades entre campos de conocimiento, con relativa autonomía, es un proceso estrechamente vinculado a las características de las áreas de conocimiento en cuestión. La sistematización e institucionalización de los estudios de la información y la comunicación se han caracterizado, entre otros aspectos, por definir: objetos de estudio, identificación disciplinaria, concepciones teóricas subyacentes, construcción histórica. Por tanto, la búsqueda de relaciones entre estos espacios se hace posible desde esas y otras dimensiones.

Pese al debate existente sobre el fenómeno de la interdisciplinariedad y su alcance, hay consenso entre los expertos en enfatizar que un rasgo distintivo de la misma: es la imprescindible interacción e intercambio de conceptos, teorías, prácticas y metodologías entre los campos de conocimiento. No obstante, el desarrollo histórico de la Ciencia de la Información y de la Ciencia de la Comunicación muestra que:

[...] El fenómeno de la importación terminológica, muy frecuente en Ciencia de la Información, devela un área que mantiene interfaces bastante próximas con diversas áreas de conocimiento, pero también permite reflexionar sobre las razones de ausencia de "exportaciones" de términos de la Ciencia de la Información para otras áreas... La elevada proporción de "nociones prestadas" es reveladora, de una interdisciplinariedad formal, que no refleja una interdisciplinariedad real, sino un préstamo de términos de otras áreas [...] (SMIT; TÁLAMO; KOBASHI, 2004). 
Esta atinada observación de los autores citados, nos coloca ante la necesaria cautela que debe tenerse en torno a las relaciones interdisciplinares entre la Ciencia de la información y la Ciencia de la Comunicación.

En esa misma línea de pensamiento, se debe anotar que son múltiples las afirmaciones de diversos autores, ya citados, de la Ciencia de la Información que contemplan a la Comunicación o a la Ciencia de la Comunicación como una de las áreas con la que se relaciona. Pero, también es notable la ausencia de autores desde la Ciencia de la Comunicación que hagan la misma consideración; en resumen, la invisibilidad de la Ciencia de la Información para la Ciencia de la Comunicación es un hecho tangible. Por todo ello, las consideraciones que siguen serán, inicialmente, una delimitación o detección de los lugares de encuentro de los cuerpos de conocimientos que se examinan. En consecuencia, será:

- Desde la Historia

El cambio epocal que se gesta y desarrolla posterior a la 2 da guerra mundial se materializa en múltiples dimensiones de la sociedad, la cultura, la política, la economía, la ciencia y la tecnología; este nuevo tiempo histórico va a generar cambios informacionales y comunicacionales que pueden calificarse como excepcionales. Son estos los tiempos de los crecimientos exponenciales de la información, de la aparición de las tecnologías computacionales, de la explosión mediática, entre otros; y serán estas algunas de las condicionantes que explican la creación formal y casi simultánea de la Ciencia de la Información y la Ciencia de la Comunicación en esa coyuntura. Con otras palabras, ambos espacios de conocimiento se formalizan e institucionalizan producto de las mismas circunstancias históricas y creándose, entre ellos, una especie de cercanía fundacional.

- Desde el objeto de estudio

La Ciencia de la Información y la Ciencia de la Comunicación, en líneas generales, son comúnmente identificadas como áreas de estudio que tienen, en un caso a la información como objeto de estudio y en otro, a la comunicación como objeto de estudio. La delimitación de la información y la comunicación como objetos de estos espacios, es también una muestra de las proximidades registrables. Se afirma, que: 
Información y ser humano son dos realidades inseparables que no pueden existir la una sin la otra. Por un lado, decir "información" implica reconocer un ser humano que la creó; y por otro lado, el estar frente a un ser humano presupone la existencia de informaciones gracias a las cuales se forma, se comunica y vive en sociedad [...]. (RENDÓN, 2017, p. 293).

Esto evidencia que el nexo existente entre Ciencia de la Información y Ciencia de la Comunicación parte, sustancialmente, del objeto información, siendo esto el eje central que las acerca.

\section{- Desde la caracterización}

La caracterización más notable recibida por la Ciencia de la Información y la Ciencia de la Comunicación es la de ser interdisciplinarias. En otro momento, de este texto se han anotado algunas consideraciones sobre este particular. No obstante, es importante subrayar que pese los debates que existen sobre este aspecto en uno y otro terreno, la interdisciplinariedad como componente intrínseco a estas áreas de estudio no parecen tener dudas; pese al limitado o inconcluso carácter que puede tener en estas materias. La interdisciplinariedad, es un punto común entre la Ciencia de la Información y la Ciencia de la Comunicación. Ellas han necesitado dialogar con otros dominios de conocimiento con el fin de elaborar sus teorías, metodologías y prácticas. Ello no excluye la no siempre explicitada relación interdisciplinaria entre la Ciencia de la Información y la Ciencia de la Comunicación.

- Desde las teorías

Las trayectorias epistemológicas de la Ciencia de la Información y de la Ciencia de la Comunicación son también lugares de encuentro entre estos campos. Una de las más relevantes coincidencias está referida a los basamentos teóricos de estos espacios en uno u otro momento de sus desarrollos. Estas áreas de estudio utilizaron como fundamento inicial de su armazón teórica, la denominada Teoría Matemática de la Comunicación de C. Shanon y W. Weaver y se expresaron en uno y otro terreno como: "paradigma físico" y "paradigma informacional".

La Ciencia de la Información bajo la impronta del Paradigma Físico se explicita en que la elaboración de sus conceptos, teorías asumidas y prácticas postuladas traducen la sustancia de ese enfoque paradigmático. El rasgo identificador de este paradigma no es otro que la creencia en la posibilidad de un conocimiento objetivo y verdadero, en tanto se pretendía crear un tipo de ciencia acorde con los postulados de la modernidad y sus Inf. Pauta, Fortaleza, CE, v. 3, número especial, nov. 2018| ISSN 2525-3468 
criterios de cientificidad. Por ello, se asume que el criterio distintivo de este paradigma no es otro que la objetividad. Por lo tanto la Ciencia de la Información, estructurada desde las posiciones de la objetividad, se apoya en la creencia de que su basamento teórico era la Teoría Matemática de la Comunicación de C. Shannon y W. Weaver. Ese intento de sustentación teórica fue infructuoso; ante todo por lo inadecuado de la concepción teórica elegida a las realidades del campo informacional (LINARES, 2005).

La Ciencia de la Comunicación, por su parte, desde el denominado paradigma informacional sustentado en el modelo desarrollado por Claude Shannon y Warren Weaver dentro de la denominada teoría matemática de la comunicación o teoría de la información, sería el más representativo de esta tendencia, que trascendió la influencia original del esquema para convertirse en un paradigma dominante durante muchos años. Es una de las primeras sistematizaciones del proceso comunicativo a partir de una perspectiva puramente técnica, cuantitativa. Desde esta mirada la comunicación es entendida como un proceso de transmisión de un mensaje por una fuente de información, a través de un canal, para un destinatario. Calificado por los estudiosos de la Ciencia de la Comunicación como una concepción simplista y reductora de los procesos comunicacionales (ALONSO; SALADRIGAS, 2006).

Las anotaciones anteriores apuntan esencialmente a la especie de sintoníaconvergencia que existe entre estas áreas de estudio en las dimensiones expuestas, pero estas cercanías no necesariamente indican la existencia de una relación interdisciplinaria, tal y como estas deben ser entendidas.

No obstante, es posible registrar determinadas experiencias interdisciplinarias entre estos espacios. Uno de los proyectos postulado por sus creadores como interdisciplinar, es el establecimiento en Francia desde mediados de los años 70 del siglo pasado de la denominada "Ciencias de la Información y la Comunicación". Se enuncia que:

[...] ¿Cómo puede preverse un estudio de la información que excluya el fenómeno de la comunicación que lo acompaña? ¿Cómo puede estudiarse el contenido de un mensaje sin considerar a los que lo crearon, a los que lo transformaron y a los que lo usan? Tales acciones, son actividades de comunicación. (IBEKWE-SANJUAN, 2012).

Estas interrogantes están en los fundamentos de esta intención interdisciplinar; sola que su materialización ha sido notablemente dificultosa. 
La institucionalización de la denominada "Ciencias de la Información y la Comunicación" plasmada en la creación de organizaciones gremiales y programas de formación, surgió y se ha desarrollado con un marcado desbalance entre las áreas de la información y la comunicación, donde esta última es la tónica distintiva y dominante en el campo creado; la denominación de interdisciplinaria que califica este espacio, mantiene la unilateralidad que hemos descrito en otros momentos. Resumiendo: "En rigor, no se encuentra en la paleta de disciplinas que conforman a las Ciencias de la Información y la Comunicación, una Ciencia de la Información bien delimitada como campo científico propio." (SILVA, 2009, p. 16).

Por último, en décadas recientes en el escenario académico se registra con relativa frecuencia la presencia de un término, infomunicación, como expresión de un "nuevo" fenómeno que pretende marcar la interacción existente entre la información y la comunicación.

Desde la Ciencia de la Información han surgido posturas que proponen un análisis de los conceptos operativos de información y comunicación, a partir de los cuales se propone una plataforma conceptual que apuesta por el entendimiento de estas categorías en una óptica complementaria y arraigada a orientaciones socioculturales. Uno de los autores más notables en este sentido, es el investigador y profesor portugués Armando Malheiro da Silva, que sostiene que la información y la comunicación representan:

[...] dos conceptos operativos que sirven para designar y comprender un fenómeno humano y social, que consiste en la capacidad innata y adquirida de dar forma (ideas, sensaciones, emociones, etc.) y de interactuar con los otros o de poner en común aquello a lo que se da forma [...]. (SILVA, 2009, p. 30).

Este autor, consecuente con esta realidad propone que la Ciencia de la Información tiene una marcada relación interdisciplinaria con la Ciencia de la Comunicación, cuya expresión más significativa es que el objeto de este campo no es otro que el fenómeno infocomunicacional, entendido como proceso que relaciona a la información con la comunicación (SILVA, 2009).

La infomunicación ha sido abordada por otros autores, así, se afirma que:

[...] Las áreas de la Ciencia de la Información, Comunicación y Ciencias de la Computación pueden ser caracterizadas como un triángulo disciplinar altamente dependiente del nuevo orden tecnocultural, tendiendo a generar la formación de una disciplina con características transdisciplinares, como infocomunicacional [...] (PINHEIRO, 2005, p. 40). 
Otros, consideran que el actual ambiente digital es el estado "ideal" del fenómeno infocomunicacional, ante la ineludible simultaneidad de la existencia, circulación y transferencia de información y comunicación (PASSARELLI, 2014).

\section{CONCLUSIONES}

La Ciencia de la Información y la Ciencia de la Comunicación en sus procesos de fundación y continuidad han buscado legitimarse como cuerpos de conocimiento, donde su caracterización como interdisciplinarias ha sido una nota distintiva. La teoría y práctica interdisciplinaria es uno de los elementos distintivos del quehacer intelectual de nuestro tiempo. Los estudios informacionales y comunicacionales tienen que ser por las peculiaridades de sus objetos y temáticas necesariamente interdisciplinares. Pero, las autodefiniciones interdisciplinarias que postulan ambos campos han estado carentes de la dimensión dialógica propia de estos processos.

Las relaciones interdisciplinarias existentes entre la Ciencia de la Información y la Ciencia de la Comunicación, pese a especificas convergencias históricas, teóricas y prácticas entre estos campos han adolecido de una autentica interdisciplinariedad; esta restricción no ha impedido que desde la Ciencia de la Información se hayan producido diversas importaciones teóricas, conceptuales y practicas provenientes de la Ciencia de la Comunicación, desde esta última esto ha sido bastante limitado.

\section{REFERÊNCIAS}

ALONSO, M.; Saladrigas, H. Teoría de la

Comunicación: una introducción a su estudio. La Habana: Editorial Pablo de la Torriente, 2006.

ARAÚJO. C. A. Á. Fundamentos da Ciência da Informação: correntes teóricas e o conceito de informação. Perspectivas em Gestão \& Conhecimento, João Pessoa, v. 4, n. 1, p. 5779, jan./jun. 2014. Disponível em: <http://periodicos.ufpb.br/ojs/index.php/p gc/article/viewFile/19120/10827>. Acesso: 23 ago. 2018.
BORKO. H. Information science: what is it? Journal of the Association for Information Science and Technology, [S.I], v. 19, n. 1, p. 3-5, 1968.

FRANÇA, V. V. Paradigmas da

Comunicação: conhecer o quê? Ciberlegenda, Niterói, n. 5, 2001. Disponível em: <https://bit.ly/2Qeu3Gs>. Acesso em: 5 ago. 2018.

GONZÁLEZ DE GOMEZ, M. N. Para uma reflexão epistemológica acerca da Ciência da Informação. Perspectivas em Ciência da 
Informação, Belo Horizonte, v. 6, n. 1, p. 518, 2001.

GUZMÁN GÓMEZ, M. El fenómeno de la interdisciplinariedad en la ciencia de la información: contexto de aparición y posturas centrales. Acimed, Cuba, v. 13, n. 3, 2005. Disponível em:

$<$ http://www.bvs.sld.cu/revistas/aci/vol13_ 3_05/aci05305.pdf>. Acesso em: 26 ago. 2018.

IBEKWE-SANJUAN F. The French conception of Information Science: une exception française?, Journal of the American Society for Information Science, [S.I], v. 63, n. 9, p. 1693-1709, 2012. Disponível em: $<$ https://onlinelibrary.wiley.com/doi/full/1 0.1002/asi.22670> . Acesso em: 27 jul. 2018.

LE COADIC. Y-F. A ciência da informação. Brasília: Briquet de Lemos, 1996.

LINARES COLUMBIÉ, R. El proyecto interdisciplinario de la Ciencia de la Información y sus primeros pasos.

Bibliotecas Anales de Investigación, Cuba, v. 13, n. 1, 2017.

\section{LINARES COLUMBIÉ. Ciencia de la} información: su historia y epistemología. Bogotá: Editorial Rojas Eberhard, 2005.

MIKHAILOV, A. I.; CHERNYI, A. I.; GILYAREVSKYI, R. S. Informatics: its scope and methods. In: MIKHAILOV, A. I.; CHERNYI, A. I.; GILYAREVSKYI, R. S. On theoretical problems of informatics. FID/ Comitê de Estudo sobre Pesquisa de Base teórica da informação, Moscou, 1969. p. 7-24.

PASSARELLI, B. et al. e-Infocomunicação: estratégias e aplicações. São Paulo: Editora Senac, 2014.

PINHEIRO, L. V. R. Campo interdisciplinar da ciência da informação: fronteiras remotas e recentes. In: PINHEIRO, L. V. R. Ciência da informação, ciências sociais e interdisciplinaridade. Brasília, Rio de Janeiro: IBICT/DDI/DEP, 1999. p. 155-182.

PINHEIRO, L. V. R. Campo interdisciplinar da Ciência da Informação: fronteiras remotas e recentes. Investigación bibliotecológica, Mexico, v. 12, n. 25, 2009.
PINHEIRO, L. V. R. Ciência da informação: desdobramentos disciplinares, interdisciplinaridade e transdisciplinaridade In: GONZALES DE GOMEZ, M. N.; DILL ORRICO, E. G. Políticas de memória e informação: reflexão na organização do conhecimento. Natal: EDUFRN, 2006. p. 111142.

PINHEIRO, L. V. R. Processo evolutivo e tendências contemporâneas da Ciência da Informação. Informacao \& Sociedade, João Pessoa, v. 15, n. 1, p. 13-48, 2005.

POMBO, 0. Contribuição para um vocabulário sobre interdisciplinaridade. In: POMBO, O; GUIMARÃES, H.; LEVY, T.

Interdisciplinaridade: reflexão e experiência. 2. ed. rev. aum. Lisboa: Texto, 1994. 102 p.

POMBO, O. Epistemologia da Interdisciplinaridade. In: SEMINÁRIO INTERNACIONAL INTERDISCIPLINARIDADE, HUMANISMO, UNIVERSIDADE, 2003, Porto. Anais... Porto, 2003. p. 1-29. Disponível em: <http://www.uesc.br/cpa/artigos/epistemo logia_interdidciplinaridade.pdf $>$. Acesso em: 11 ago. 2018.

POMBO, O. Interdisciplinaridade e integração dos saberes. Liinc em revista, Rio de Janeiro, v. 1, n. 1, 2005. Disponível em: <http://revista.ibict.br/liinc/article/viewFil e/3082/2778>. Acesso em: 10 ago. 2018.

RENDÓN, R, M. A. La información y la dialéctica del desarrollo del ser humano. Inf. Inf, Londrina, v. 22, n. 2, p. 293 - 319, maio/ago., 2017. Disponível em: <http:www.uel.br/revistas/informacao/>. Acesso em: 22 ago. 2018.

SARACEVIC, Tefko. Ciência da informação: origem, evolução e relações. Perspectivas em Ciência da Informação, Belo Horizonte, v. 1, n. 1, p. 41-62, jan/jun, 1996.

SARACEVIC, Tefko. Information science.

Journal of American Society for Information Science, Washington, v. 50, n. 12, p. 1051-1063, 1999.

SARACEVIC, Tefko. Information Science: origin, evolution and relations. In: VAKKARI, P.; CRONIN, B. (Ed.). Conceptions of library Inf. Pauta, Fortaleza, CE, v. 3, número especial, nov. 2018 | ISSN 2525-3468 
and Information Science: historical, empirical and theoretical perspectives.

Proceedings of the International Conference for the celebration of 20th anniversary of the Department of Information Studies. London: Taylor Grahan, 1992, p. 5-27.

SILVA, A. Informação y comunicação como projecto epistemológico em Portugal e no Brasil. In: SILVA, A. A medicina na era da informação. Salvador: EDUFBA. 2009.
SMIT, J. W.; TÁLAMO, M. F.; KOBASHI, N. A determinação do campo científico da ciência da informação: uma abordagem terminológica. Datagramazero, Rio de Janeiro, v. 5, n. 1, 2004. Disponível em: <http://www.brapci.ufpr.br/brapci/_reposit orio/2010/01/pdf_5d8bd56608_0007603.p df >. Acesso em: 25 jul. 2018.

ZINS, C. Conceptions of information science. Journal of the American Society for Information Science and Technology, v. 58, n. 3, p. 335-350, 2007.

\section{NOTAS}

i A revisão ortográfica, gramatical e em Língua Vernácula é de responsabilidade do autor.

ii La primera definición de Ciencia de la Información le cupo a Robert Taylor, en tres variantes, publicadas en 1963 y 1966. Harold Borko, sintetiza las variantes de Taylor en un artículo publicado en 1968 y considerado con justeza como un clásico, Information Science, what is it? 